\title{
HIV Vaccine Research Agenda: Perspectives of an HVTN Site Investigator William A Blattner*
}

Address: IHV, UMD, Baltimore, MD 21201

Email: William A Blattner* - blattner@umbi.umd.edu

* Corresponding author $\ddagger$ Presenting author

from 2005 International Meeting of The Institute of Human Virology

Baltimore, USA, 29 August - 2 September 2005

Published: 8 December 2005

Retrovirology 2005, 2(SuppI I):S38 doi:I0.II86/I742-4690-2-SI-S38

The HIV Vaccine Trials Network has as its central mission, the implementation of clinical trials of candidate vaccine products (immunogens, vector, delivery, adjuvants) that induce immunologic responses relevant to preventing HIV infection and/or controlling HIV disease progression. The research agenda targets evaluation of candidates at all stages of product development from phase 1 first in human trials to proof of concept and efficacy trials. Innovative trial designs are driven by investigators and statisticians to efficiently advance products and information that seeks fundamental insight into correlates of protective immunity. HVTN laboratories quantitate adaptive and innate immune responses employing highly reproducible and standardized endpoint assays that meet GLP validation standards for core assessment as well as exploratory assays to understand correlate mechanisms. Currently 24 HIV vaccine candidates are slated for evaluation over the next 24 months including proof of concept and expanded phase 2 evaluation in preparation for larger efficacy trials in the 2007-2008 time period. 